\title{
Combinatorial proof for a stability property of plethysm coefficients
}

\author{
Laura Colmenarejo Hernando ${ }^{1,2}$ \\ Departamento de Álgebra \\ Universidad de Sevilla \\ Sevilla, Spain \\ Emmanuel Briand ${ }^{1,3}$ \\ Departamento de Matemática Aplicada I \\ Universidad de Sevilla \\ Sevilla, Spain
}

\begin{abstract}
Plethysm coefficients are important structural constants in the representation theory of the symmetric groups and general linear groups. Remarkably, some sequences of plethysm coefficients stabilize (they are ultimately constants). In this paper we give a new proof of such a stability property, proved by Brion with geometric representation theory techniques. Our new proof is purely combinatorial: we decompose plethysm coefficients as a alternating sum of terms counting integer points in polytopes, and exhibit bijections between these sets of integer points.

Keywords: Combinatorial representation theory, symmetric functions, plethysm.

1 Supported by project MTM2010-19336 and FEDER, and Junta de Andalucia under grants FQM-333 and P12-FQM-2696.

2 Email: laurach@us.es

3 Email: ebriand@us.es
\end{abstract}




\section{Introduction}

Representation theory of groups is a fundamental tool in geometry and mathematical physics. But even one of the simplest cases, the representation theory of the general linear groups $G L_{n}(\mathbb{C})$, still raises unsolved problems. It is known that any (finite-dimensional, complex, analytic) linear representation $V$ of $G L_{n}(\mathbb{C})$ decomposes as a direct sum of irreducible representations:

$$
V \approx \bigoplus_{\lambda} m_{\lambda} S_{\lambda}\left(\mathbb{C}^{n}\right)
$$

Here the $m_{\lambda}$ are nonnegative integers (the multiplicities of the irreducible representations in $V)$. The irreducible representations $S_{\lambda}\left(\mathbb{C}^{n}\right)$, indexed by the integer partitions $\lambda$ of length at most $n$ (an integer partition of length $k$ is a weakly decreasing sequence of $k$ positive integers), can be built explicitly by means of explicit combinatorial constructions $[7,6]$. These constructions can be applied actually to any representations: the $S_{\lambda}$ are actually endofunctors of the category of representation of $G L_{n}(\mathbb{C})$ (the Schur functors) that comprise as particular cases the symmetric powers and the exterior powers.

Three important, non-trivial, constructions of new representations from old ones are: tensor products; restrictions to a subgroup; plethysms (composition of functors $S_{\lambda}$ ). They define three important families of structural constants. Firstly, the Littlewood-Richardson coefficients $c_{\mu, \nu}^{\lambda}$ are the multiplicities arising when decomposing a tensor product of irreducible representations $S_{\mu}\left(\mathbb{C}^{n}\right) \otimes S_{\nu}\left(\mathbb{C}^{n}\right)$. Next, the Kronecker coefficients $g_{\lambda}^{\mu, \nu}$ are the multiplicities arising when considering an irreducible representation of $G L_{m n}(\mathbb{C})$ as a representation of $G L_{m}(\mathbb{C}) \times G L_{n}(\mathbb{C})$ by means of the Kronecker product of matrices, and decomposing it into irreducibles. Finally, the plethysm coefficients $a_{\pi, \nu}^{\lambda}$ are the multiplicities obtained when applying a Schur functor $S_{\pi}$ to an irreducible representation $S_{\nu}\left(\mathbb{C}^{n}\right)$ and decomposing the resulting representation in irreducibles.

The Littlewood-Richardson coefficients are quite well understood: they have many combinatorial interpretations (they count Littlewood-Richardson Young tableaux, or alternatively the integral points in the hive polytopes) that are as many efficient tools for proofs and as well for computations. Finding similar general interpretations for the Kronecker coefficients and the plethysm coefficients are major open problems in combinatorial representation theory.

Murnaghan [10] and Littlewood [8] observed some remarkable stability properties of the Kronecker coefficients: some sequences of Kronecker coefficients are ultimately constants. Proofs for these stability properties were 
provided by Thibon and his his collaborators by means of vertex operators on symmetric functions [11] and by Brion by means of tools from geometric representation theory [1]. Very recently a new proof were provided by Church, Farb and Ellenberg by means of explicit constructions of sequences of representations of symmetric groups in the setting of their theory of FI-modules [4], at the same time they observed the ubiquity of the phenomenon of stability of multiplicities.

The plethysm coefficients also exhibit several stability properties, some observed by Foulkes [5], that were proved in the 1990's essentially by means of combinatorial arguments (vertex operators and symmetric functions) by Carré and Thibon [2] on the one hand, and by tools from geometric representation theory by Brion on the other hand [1] in a more general setting (algebraic groups in general rather than just general linear groups). Some of the stability properties proved by Brion lack combinatorial proofs. In this paper we consider one of them.

Theorem 1.1 (Brion [1]) For any partitions $\pi, \lambda$ and $\mu$ such that $|\lambda| \cdot|\pi|=$ $|\mu|$, the sequence with general term $u_{n}=a_{\pi+(n), \lambda}^{\mu+n \lambda}$ stabilizes.

Here for any partition $\alpha=\left(\alpha_{1}, \alpha_{2}, \ldots, \alpha_{k}\right)$, the notation $|\alpha|$ stands for the sum of its parts,

$$
|\alpha|=\alpha_{1}+\alpha_{2}+\cdots+\alpha_{k}
$$

and we treat partitions as vectors: we can addi them, and multiply them by scalars (e.g. in $\mu+n \lambda$ ), adding trailing zeros whenever necessary (e.g adding two partitions of different lengths).

Our contribution is a new, simple, combinatorial proof of Theorem 1.1, that we sketch now. We set the plethysm coefficients $a_{\pi, \lambda}^{\mu}$ in the framework of symmetric functions and express them as alternated sums of coefficients $b_{\pi+\omega, \lambda}^{\mu+\omega^{\prime}}$. The stability of the sequence coefficients $a_{\pi+(n), \lambda}^{\mu+n \lambda}$ will therefore be established as soon as all sequences of coefficients $b_{\pi+\omega+(n), \lambda}^{\mu+n \lambda \omega^{\prime}}$ are shown to be stable.

We show that the coefficient $b_{\pi, \lambda}^{\mu}$ counts the integer points in a polytope $Q(\pi, \lambda, \mu)$. Let $E(n)$ stand for $Q(\pi+(n), \lambda, \mu+n \lambda)$. We build explicit injections $E(n) \hookrightarrow E(n+1)$. Last we show that these injections are surjective for $n$ big enough. 


\section{From plethysm coefficients to integer points in poly- topes}

The (complex, analytic, finite-dimensional) linear representations of $G L(n, \mathbb{C})$ are completely described, up to isomorphism, by their character, which is a symmetric polynomial $[7,6]$. This allows to set computations of plethysms in the framework of symmetric functions. Remember (see for instance $[9,12,6]$ ) that the ring of symmetric functions is a graded ring endowed with a scalar product, that admits several importat linear basis. Among them: the Schur functions $s_{\lambda}$, the monomial functions $m_{\lambda}$, and the products of complete functions $h_{\lambda}=h_{\lambda_{1}} h_{\lambda_{2}} \cdots h_{\lambda_{k}}$. All these bases are indexed by the integer partitions $\lambda$ (the complete sums $h_{k}$ are indexed by the nonnegative integers and as a result the products of complete sums are also indexed by integer partitions). With respect to the scalar product, the Schur functions are an orthonormal basis. The monomial functions $m_{\lambda}$ and the complete sums $h_{\lambda}$ are dual bases.

The plethysm of representations induces an operation $(f, g) \mapsto f[g]$ on the ring of symmetric functions, called plethysm of symmetric functions (see [9], I.8). This operation is associative but non-commutative, and not even bilinear. It is, nevertheless, linear in the first argument. In this framework, the plethysm coefficient $a_{\mu, \nu}^{\lambda}$ is the coefficient of $s_{\lambda}$ in the expansion in the Schur basis of the plethysm of Schur functions $s_{\mu}\left[s_{\nu}\right]$. Alternatively, this coefficient is extracted by means of a scalar product:

$$
a_{\mu, \nu}^{\lambda}=\left\langle s_{\mu}\left[s_{\nu}\right] \mid s_{\lambda}\right\rangle
$$

We will expand, in this expression, $s_{\mu}$ and $s_{\lambda}$ in the $h$-basis. This is done by means of the Jacobi-Trudi identity that we recall here.

Lemma 2.1 (Jacobi-Trudi identity, [9] I. (3.4)) Let $\lambda$ be a partition with length at most $N$. Then

$$
s_{\lambda}=\operatorname{det}\left(h_{\lambda_{j}+i-j}\right)_{1 \leq i, j \leq N}
$$

with $h_{0}=1$ and $h_{r}=0$ if $r<0$, and $\lambda$ is completed with trailing zeros if necessary.

This expansion writes explicitly as a sum over the permutations $\sigma$ in the symmetric group $\mathfrak{S}_{N}\left([9]\right.$ I. $\left.\left(3.4^{\prime}\right)\right)$

$$
s_{\lambda}=\sum_{\sigma \in \mathfrak{S}_{N}} \varepsilon(\sigma) h_{\lambda+\omega(\sigma)}
$$


where $\omega(\sigma)_{j}=\sigma(j)-j$ for all $j$ between 1 and $N$.

We now perform this Jacobi-Trudi expansion for $s_{\mu}$ and $s_{\nu}$ in (1). We get the following expansion for the plethysm coefficients.

Lemma 2.2 Let $N$ and $N^{\prime}$ be positive integers. Let $\lambda, \mu$ and $\nu$ be partitions, such that $\mu$ has length at most $N$ and $\lambda$ has length at most $N^{\prime}$. Then

$$
a_{\mu, \nu}^{\lambda}=\sum_{\sigma, \tau} \varepsilon(\sigma) \varepsilon(\tau)\left\langle h_{\mu+\omega(\sigma)}\left[s_{\nu}\right] \mid h_{\lambda+\omega(\tau)}\right\rangle
$$

where the sum is carried over all permutations $\sigma \in \mathfrak{S}_{N}$ and $\tau \in \mathfrak{S}_{N^{\prime}}$.

The scalar products that appear on the right-hand side are interesting. For any partition $\nu$ and any finite sequences $\mu$ and $\lambda$ of integers we set:

$$
b_{\mu, \nu}^{\lambda}=\left\langle h_{\mu}\left[s_{\nu}\right] \mid h_{\lambda}\right\rangle \text {. }
$$

It turns out that these coefficients count the nonnegative solutions of systems of linear Diophantine equations whose constant terms depend linearly on the parts of $\mu$ and $\lambda$. In particular, they count integer points in polytopes with a nice description.

To state this precisely we introduce some notations. For any partition $\nu$ and any positive integer $N$ let $t(\nu ; N)$ be the set of semi-standard Young tableaux (see [12] 7.10) of shape $\nu$ with entries between 1 and $N$. Let $\mathcal{P}_{\nu ; N}=$ $\left(p_{T, j}\right)_{T, j}$ be the matrix whose rows are indexed by the tableaux $T \in t(\nu ; N)$, whose columns are indexed by the integers $j$ between 1 and $N$, such that $p_{T, j}$ is the number of occurrences of $j$ in $T$ (so that row $T$ of $\mathcal{P}_{\nu ; N}$ is the weight of the tableau $T$ ).

Proposition 2.3 Let $\mu, \nu$ and $\lambda$ be partitions. Let $\ell(\mu)$ be the length of $\mu$ and $N$ be at least the length of $\lambda$.

The coefficient $b_{\mu, \nu}^{\lambda}$ is the cardinal of the set $Q(\mu ; \nu ; \lambda ; N)$ of matrices $\mathcal{M}=$ $\left(m_{i, T}\right)$ with nonnegative integer entries whose rows are indexed by the integers $i$ between 1 and $\ell(\mu)$ and whose columns are indexed by the tableaux $T \in t(\nu ; N)$ such that:

- The sum of the entries in row $i$ of $\mathcal{M}$ is $\mu_{i}$.

- The sum of the entries in column $j$ of $\mathcal{M} \cdot \mathcal{P}_{\nu ; N}$ is $\lambda_{j}$.

Proof. [Sketch] Let $x_{1}, x_{2}, \ldots$ be the underlying variables of the symmetric functions and, for any finite sequence $\lambda=\left(\lambda_{1}, \lambda_{2}, \ldots, \lambda_{k}\right)$, let $x^{\lambda}=$ $x_{1}^{\lambda_{1}} x_{2}^{\lambda_{2}} \cdots x_{k}^{\lambda_{k}}$. 
Since the scalar product with $h_{\lambda}$ extracts the coefficient of $m_{\lambda}$ in the expansion in basis of monomial functions, the constant $b_{\mu, \nu}^{\lambda}$ can be interpreted as the coefficient as the monomial $x^{\lambda}$ in $h_{\mu}\left[s_{\nu}\right]$. Instead of working with symmetric functions (with infinitely many variables) we can work with symmetric polynomials in $N$ variables, provided $N$ is at least the length of $\lambda$. We now use the expansion of the Schur polynomial $s_{\nu}\left(x_{1}, x_{2}, \ldots, x_{N}\right)$ in monomials ([6]):

$$
s_{\nu}\left(x_{1}, x_{2}, \ldots, x_{N}\right)=\sum_{T \in t(\nu ; N)} x_{1}^{p_{1, T}} x_{2}^{p_{2, T}} \cdots x_{N}^{p_{N, T}} .
$$

We also use that a plethysm $f[g]$ when $g$ is a sum of monomials $g=\sum_{i} x^{u_{i}}$ is just the evaluation $f\left(x^{u_{1}}, x^{u_{2}}, \ldots\right)$ (see [12] 7.8). We use finally that the complete sum $h_{j}$ is the sum of all monomials of degree $j$. These three facts and a short combinatorial reasoning provide the expansion:

$$
h_{\mu}\left[s_{\nu}\left(x_{1}, \ldots, x_{N}\right)\right]=\sum_{\lambda} \operatorname{Card}(Q(\mu ; \nu ; \lambda ; N)) x^{\lambda} .
$$

This proves the proposition.

\section{Stability}

We now consider a positive integer $N$ and three partitions $\lambda, \pi$ and $\mu$, where $\lambda$ and $\mu$ have length at most $N$ and $|\lambda| \cdot|\pi|=|\mu|$. Applying Lemma 2.2 to the plethysm coefficients $u_{n}=a_{\pi+(n), \lambda}^{\mu+n \lambda}$ we get

$$
u_{n}=\sum_{\sigma \in \mathfrak{S}_{\ell(\pi)}, \tau \in \mathfrak{S}_{N}} \varepsilon(\sigma) \varepsilon(\tau) v_{n}(\sigma, \tau)
$$

where $v_{n}(\sigma, \tau)=b_{\pi+\omega(\sigma)+(n), \lambda}^{\mu+n \lambda+\omega(\tau)}$.

Therefore, in order to establish Theorem 1.1 (the stability of the sequence with general term $u_{n}$ ), it is enough to show that each of the sequences $v_{n}(\sigma, \tau)$ stabilizes. Each of these sequences is of the type $b_{\pi^{\prime}+(n), \lambda}^{\mu^{\prime}+n \lambda}$ for some sequences $\pi^{\prime}$ and $\mu^{\prime}$.

Theorem 3.1 Let $\lambda$ be a partition and $\pi$ and $\mu$ be finite sequences of integers. Then, the sequence with general term $v_{n}=b_{\pi+(n), \lambda}^{\mu+n \lambda}$ stabilizes.

Proof. [Sketch] Let $N$ be an integer bigger than or equal to the lengths of $\lambda$ 
and $\mu$. After Proposition 2.3,

$$
v_{n}=\operatorname{Card}(Q(\pi+(n) ; \lambda ; \mu+n \lambda)) .
$$

Set $E(n)$ for $Q(\pi+(n) ; \lambda ; \mu+n \lambda)$.

Let $T_{0}$ be the tableau in $t(\lambda ; N)$ whose row number $i$ is filled with occurrences of $i$, for each $i$. Consider the injection $\iota(n): E(n) \hookrightarrow E(n+1)$ that maps any matrix $\mathcal{M} \in E(n)$ to the matrix $\mathcal{M}^{\prime}$ where the coefficient $m_{1, T_{0}}$ is incremented by one, and all other coefficients are unchanged.

We contend that $\iota(n)$ is also surjective for $n$ big enough. It is not difficult to check that $\iota(n)$ is surjective if and only if for all $\mathcal{M}^{\prime} \in E(n+1)$, the entry $m_{1, T_{0}}$ is non-zero. Thus proving the surjectivity of $\iota(n)$ amounts to showing that $m_{1, T_{0}}>0$.

Let $\mathcal{M}^{\prime} \in E(n+1)$. Observe that among all tableaux in $t(\lambda ; N)$, the tableau $T_{0}$ is the unique one with maximum weight for the dominance ordering ([9] I.1). Denote $\|\alpha\|$ for $\sum_{k=1}^{N} \sum_{j=1}^{k} \alpha_{j}$ and $p_{T}$ for row $T$ of $\mathcal{P}_{\lambda ; N}$. Then for all $T \neq T_{0}$,

$$
\left\|p_{T}\right\| \leq\|\lambda\|-1
$$

and for $T=T_{0}$,

$$
\left\|p_{T_{0}}\right\|=\|\lambda\| \text {. }
$$

Using now the row sums conditions on the matrix $\mathcal{M}^{\prime}$, a few more elementary operations lead to the inequality

$$
m_{1, T_{0}} \geq\|\mu\|+\pi_{1}-|\pi| \cdot|| \lambda||+(n+1)
$$

that proves that $m_{1, T_{0}}>0$ as soon as $n \geq|\pi| \cdot|| \lambda||-|| \mu||-\pi_{1}$.

\section{Conclusion}

We have provided with rather elementary tools (the combinatorics of symmetric functions) a new proof of a stability property for plethysm coefficients, otherwise proved using the more eleborate machinery of geometric representation theory. Our approach enhanced the importance of other constants (the $b_{\lambda, \mu}^{\nu}$ in our paper) that seem interesting by themselves. In particular, since they count integer points in polytopes, it should be possible to evaluate them efficiently by means of Barvinok's algorithm. Does this lead to more efficient algorithms for computing the plethysm coefficients? A similar approach was followed successfully for the Kronecker coefficients [3]. 


\section{References}

[1] Brion, Michel, Stable properties of plethysm: on two conjectures of Foulkes. Manuscripta Math. 80(4) (1993), 347-371.

[2] Carré, Christophe and Jean-Yves Thibon, Plethysm and vertex operators, Adv. in Appl. Math., 13 (4) (1992), 390-403.

[3] Christandl, M., B. Doran and M. Walter, Computing multiplicities of Lie group representations. In Foundations of Computer Science (FOCS), 2012 IEEE 53rd Annual Symposium on, pages 639-648, Oct 2012.

[4] Church, Thomas, Jordan Ellenberg and Benson Farb., Fi-modules: a new approach to stability for $S_{n}$-representations. arXiv:1204.4533[math.RT], april 2012.

[5] Foulkes, H. O., Concomitants of the quintic and sextic up to degree four in the coefficients of the ground form. J. London Math. Soc. 25 (1950), 205-209.

[6] Fulton, William, "Young tableaux", volume 35 of London Mathematical Society Student Texts. Cambridge University Press, Cambridge, 1997. With applications to representation theory and geometry.

[7] Fulton, William and Joe Harris, Representation theory, volume 129 of Graduate Texts in Mathematics. Springer-Verlag, New York, 1991. A first course, Readings in Mathematics.

[8] Littlewood, D. E., The Kronecker product of symmetric group representations. J. London Math. Soc., 31 (1956), 89-93.

[9] Macdonald, I. G., "Symmetric functions and Hall polynomials". Oxford Mathematical Monographs. The Clarendon Press, Oxford University Press, New York, second edition, 1995. With contributions by A. Zelevinsky, Oxford Science Publications.

[10] Murnaghan, Francis D., The Analysis of the Kronecker Product of Irreducible Representations of the Symmetric Group, Amer. J. Math. 60 (3) (1938), 761784 .

[11] Scharf, Thomas, Jean-Yves Thibon and Brian G. Wybourne, Reduced notation, inner plethysms and the symmetric group, J. Phys. A 26 (24) (1993), 7461-7478.

[12] Stanley, Richard P., Enumerative combinatorics. Vol. 2, volume 62 of Cambridge Studies in Advanced Mathematics. Cambridge University Press, Cambridge, 1999. With a foreword by Gian-Carlo Rota and appendix 1 by Sergey Fomin. 